University of Wollongong

Research Online

Faculty of Engineering and Information

Faculty of Engineering and Information

Sciences - Papers: Part A

Sciences

$1-1-2016$

\title{
Indoor scene reconstruction for through-the-wall radar imaging using low- rank and sparsity constraints
}

Van H. Tang

University of Wollongong, vtang@uow.edu.au

Abdesselam Bouzerdoum

University of Wollongong, bouzer@uow.edu.au

Son Lam Phung

University of Wollongong, phung@uow.edu.au

Fok Hing Chi Tivive

University of Wollongong, tivive@uow.edu.au

Follow this and additional works at: https://ro.uow.edu.au/eispapers

Part of the Engineering Commons, and the Science and Technology Studies Commons

Research Online is the open access institutional repository for the University of Wollongong. For further information contact the UOW Library: research-pubs@uow.edu.au 


\title{
Indoor scene reconstruction for through-the-wall radar imaging using low-rank and sparsity constraints
}

\author{
Abstract \\ This paper addresses the problem of indoor scene reconstruction in compressed sensing through-the- \\ wall radar imaging. The proposed method is motivated by two observations that wall reflections reside in \\ a low-rank subspace and the imaged scene tends to be sparse. The task of mitigating the wall reflections \\ and reconstructing an image of the scene behind-the-wall is cast as a joint low-rank and sparsity \\ constrained optimization problem, where a low-rank matrix captures the wall returns and a sparse matrix \\ represents the formed image. An iterative algorithm is developed to estimate the low-rank matrix and the \\ sparse scene vector from a reduced measurement set. Experimental results using real radar data show \\ that the proposed model is very effective at reconstructing the indoor image and removing wall clutter.

\section{Keywords} \\ reconstruction, wall, radar, imaging, indoor, low, scene, rank, sparsity, constraints

\section{Disciplines} \\ Engineering | Science and Technology Studies

\section{Publication Details} \\ V. H. Tang, A. Bouzerdoum, S. L. Phung \& F. H. C. Tivive, "Indoor scene reconstruction for through-the-wall \\ radar imaging using low-rank and sparsity constraints," in 2016 IEEE Radar Conference (RadarConf), 2016, \\ pp. 1-4.
}




\title{
Indoor Scene Reconstruction for Through-the-Wall Radar Imaging using Low-Rank and Sparsity Constraints
}

\author{
V. H. Tang, A. Bouzerdoum, S. L. Phung, and F. H. C. Tivive \\ School of Electrical, Computer and Telecommunications Engineering, \\ University of Wollongong, NSW 2522, Australia \\ (Email:vht986@uowmail.edu.au, a.bouzerdoum@uow.edu.au,phung@uow.edu.au, tivive@uow.edu.au)
}

\begin{abstract}
This paper addresses the problem of indoor scene reconstruction in compressed sensing through-the-wall radar imaging. The proposed method is motivated by two observations that wall reflections reside in a low-rank subspace and the imaged scene tends to be sparse. The task of mitigating the wall reflections and reconstructing an image of the scene behind-the-wall is cast as a joint low-rank and sparsity constrained optimization problem, where a low-rank matrix captures the wall returns and a sparse matrix represents the formed image. An iterative algorithm is developed to estimate the low-rank matrix and the sparse scene vector from a reduced measurement set. Experimental results using real radar data show that the proposed model is very effective at reconstructing the indoor image and removing wall clutter.
\end{abstract}

\section{INTRODUCTION}

Through-the-wall radar (TWR) imaging is an emerging technology that aims to interrogate scenes behind walls and other visually opaque materials. The ability to sense through enclosed building structures is highly desirable in numerous civilian and military applications, including search-and-rescue missions and surveillance and reconnaissance operations [1], [2]. The development of efficient TWR sensing systems, however, faces several challenges. One of the major challenges is to detect stationary indoor targets for compressed sensing operations in which not all data samples can be acquired successfully, due to competing wireless services, intentional interferences, or radar jamming [3], [4]. Furthermore, the strong front-wall electromagnetic returns overwhelm the target reflections, rendering target detection very difficult, or even impossible [5]. Therefore, developing an efficient technique that can mitigate the wall clutter and form the image of the indoor targets is essential for TWR imaging.

Several imaging techniques have been proposed for TWR and urban operations. Conventional backprojection methods, such as delay-and-sum (DS) beamforming can be used to form a scene image if the full measurement set is available [6]. Compressed sensing (CS) has been employed for fast data acquisition and accurate signal reconstruction from compressed measurements [7], [8]. Many CSbased TWR imaging techniques assume that the wall returns have been removed, or measurements from the background scene are available for suppressing the wall reflections [9]-[12]. Recent CSbased methods for TWR indoor scene reconstruction consist of two stages [13]-[15]: (i) wall clutter mitigation, and (ii) image formation. Before the wall clutter mitigation stage, the antenna signals have to be recovered from reduced data samples using $\ell_{1}$ minimization [13], joint Bayesian sparse approximation [14], or block-sparse estimation [15]. Then, existing wall clutter mitigation methods, such as spatial filtering [16], or subspace projection [17], [18] are applied before image formation. The issue of these two-stage methods is that the image formation depends on the accuracy of the signal recovery and the wall clutter mitigation operations.

In this paper, we propose a new image formation technique for compressed sensing TWR imaging. The proposed approach is motivated by the observation that the wall reflections reside in a low- rank subspace and the images of indoor targets tend to be sparse. We model wall clutter mitigation and image formation as a joint low-rank and sparse constrained optimization problem. An iterative technique is developed to estimate a low-rank matrix and a sparse vector. The low-rank matrix captures the wall returns, and the sparse vector represents the image of the targets.

The remainder of the paper is organized as follows. Section II introduces the TWR signal model. Section III describes the proposed joint low-rank and sparsity model for removing the wall clutter and reconstructing an image of the behind-wall scene. Section IV presents the experimental setup and results. Section $\mathrm{V}$ gives concluding remarks.

\section{TWR SIGNAL MODEL}

Consider a monostatic stepped-frequency TWR system where a transceiver is placed at several scan positions parallel to the wall to synthesize a horizontal $M$-element linear antenna array. The scene is interrogated by transceiving a stepped-frequency signal comprising $N$ frequencies, equally spaced over the sensing bandwidth. Suppose that the scene contains $P$ targets placed behind the wall. Let $z_{n, m}$ denote the $n$-th frequency signal received at the $m$-th antenna location. The signal $z_{n, m}$ is modeled as the superposition of the wall reflections $z_{n, m}^{w}$ (including the wall reverberations), target returns $z_{n, m}^{t}$, and noise $v_{n, m}$ :

$$
z_{n, m}=z_{n, m}^{w}+z_{n, m}^{t}+v_{n, m} .
$$

The wall component $z_{n, m}^{w}$ is given by

$$
z_{n, m}^{w}=\sum_{r=1}^{R} \sigma_{w} a_{r} e^{-j 2 \pi f_{n} \tau_{m, w}^{r}},
$$

where $\sigma_{w}$ is the reflectivity of the wall, $R$ is the number of wall reverberations, $a_{r}$ is the path loss factor associated with the $r$-th wall return, and $\tau_{m, w}^{r}$ is the propagation delay of the $r$-th wall reverberation. The target return can be expressed as

$$
z_{n, m}^{t}=\sum_{p=1}^{P} \sigma_{p} e^{-j 2 \pi f_{n} \tau_{m, p}},
$$

where $\sigma_{p}$ is the reflectivity of the $p$-th target, and $\tau_{m, p}$ is the roundtrip travel time of the signal from the $m$-th antenna location to the $p$-th target.

For image formation, the behind-the-wall scene is partitioned into a rectangular grid comprising $Q$ pixels, and the image pixels are arranged into a vector $\mathbf{s} \in \mathbb{C}^{Q \times 1}$. The value $s_{q}$ can be considered as a weighted indicator function representing the target reflectivity defined as

$$
s_{q}= \begin{cases}\sigma_{p}, & \text { if the } p \text {-th target occupies the } q \text {-th pixel } \\ 0, & \text { otherwise. }\end{cases}
$$


Furthermore, a wall mitigation technique, such as spatial filtering [16] or subspace projection [5], [17], [18] is applied to the measurements to recover the target signal $z_{n, m}^{t}$ from $z_{n, m}$. Let $\tau_{m, q}$ denote the focusing delay between the $m$-th antenna and the $q$-th pixel. Assuming the target consists of points located precisely on the image pixels, from (3) the target signal at the $m$-th antenna can be expressed in matrix-vector form as

$$
\mathbf{z}_{m}^{t}=\Psi_{m} \mathbf{s}
$$

where $\mathbf{z}_{m}^{t}=\left[z_{1, m}^{t}, \ldots, z_{N, m}^{t}\right]^{T}$ and $\Psi_{m}=\left[\psi_{m}(n, q)\right]$ is an $N \times Q$ matrix with the $(n, q)$-th element given by $\psi_{m}(n, q)=$ $\exp \left(-j 2 \pi f_{n} \tau_{m, q}\right)$. The relation between the collected measurements and the formed image $\mathbf{s}$ can be expressed as

$$
\mathbf{z}^{t}=\Psi \mathbf{s},
$$

where $\mathbf{z}^{t}=\left[\left(\mathbf{z}_{1}^{t}\right)^{T}, \ldots,\left(\mathbf{z}_{M}^{t}\right)^{T}\right]^{T}, \Psi=\left[\Psi_{1}^{T}, \ldots, \Psi_{M}^{T}\right]^{T}$.

In practical compressed TWR imaging applications, only a subset of frequency samples is available at each antenna location, but for wall clutter mitigation, a full set of frequency samples or the same frequency samples at all antenna locations are often required. Therefore, many CS-based methods comprise two separate stages to form the image of behind-the-wall scene: signal reconstruction followed by wall clutter mitigation and image formation. The next section describes the proposed joint low-rank and sparsity-based model for combining wall clutter mitigation and image formation.

\section{JOINT LOW-RANK AND SPARSITY MODEL}

This section presents an image formation method using low-rank and sparsity constraints. First, the received measurements from different antennas are arranged into a matrix. Then, the task of mitigating the wall reflections and reconstructing the image of the targets is cast as a joint low-rank and sparse constrained optimization problem, where the nuclear-norm is used to enforce the low-rank property of the wall clutter and the $\ell_{1}$-norm is used to provide the sparsity of the target image. Moreover, an iterative algorithm is introduced to solve the optimization problem, yielding an image of the indoor targets.

\section{A. Problem formulation}

Let $\mathbf{Z}=\left[z_{n, m}\right], \mathbf{Z}^{w}=\left[z_{n, m}^{w}\right], \mathbf{Z}^{t}=\left[z_{n, m}^{t}\right]$, and $\mathbf{\Upsilon}=\left[v_{n, m}\right]$ denote the $N \times M$ matrices containing, respectively, the radar signals, the wall reflections, the target returns, and the noise received by all $M$ antennas for all $N$ frequencies. Equation (1) can be rewritten in matrix-form as

$$
\mathbf{Z}=\mathbf{Z}^{w}+\mathbf{Z}^{t}+\mathbf{\Upsilon}
$$

Now, the objective is to decompose the data matrix $\mathbf{Z}$ into a low-rank matrix $\mathbf{Z}^{w}$ carrying the wall returns, a sparse matrix $\mathbf{Z}^{t}$ containing the target signal, and noise. This decomposition task is known as stable principal component pursuit (SPCP) [19]. Both the low-rank and sparse matrices can be recovered by minimizing a weighted combination of the nuclear norm and $\ell_{1}$ norm. Let $\left\|\mathbf{Z}^{w}\right\|_{*}$ denote the nuclear norm (i.e. the sum of the singular values of the matrix $\mathbf{Z}^{w}$ ) and let $\left\|\mathbf{Z}^{t}\right\|_{1}$ be the $\ell_{1}$-norm of $\mathbf{Z}^{t}$. Then, the low-rank component $\mathbf{Z}^{w}$ and sparse component $\mathbf{Z}^{t}$ can be estimated by solving the following optimization problem:

$$
\begin{array}{ll}
\underset{\mathbf{Z}^{w}, \mathbf{Z}^{t}}{\operatorname{minimize}} & \left\|\mathbf{Z}^{w}\right\|_{*}+\lambda\left\|\mathbf{Z}^{t}\right\|_{1} \\
\text { subject to } & \left\|\mathbf{Z}-\left(\mathbf{Z}^{w}+\mathbf{Z}^{t}\right)\right\|_{F}^{2} \leq \epsilon,
\end{array}
$$

where $\lambda$ is a regularization parameter used to achieve a trade off between the low-rank and sparse constraints, and $\epsilon$ is a noise bound.
Algorithms including convex optimization method [20] or greedy technique [21] can be applied to solve this minimization problem. These methods, however, are either applicable for full sensing mode where all entries of $\mathbf{Z}$ are available or they mainly focus on recovering the low-rank matrix only. Note that our focus here is to estimate a sparse vector $\mathbf{s}$ representing the scene targets from a compressed measurement set. Therefore, in the next section, we extend the formulation for TWR imaging and introduce an iterative algorithm for efficient scene reconstruction and wall clutter mitigation.

\section{B. Optimization algorithm}

Since the signals reflected from the front wall are similar across the antenna array and the number of target pixels is far fewer than the total number of image pixels, the target scene reconstruction can be formulated into a similar optimization problem. In compressed sensing TWR imaging, a reduced data set of $K$ measurements is acquired $(K \ll N \times M)$. Let $\boldsymbol{\Phi}$ denote a sensing matrix in which each row has only one non-zero element indicating the selected frequency for a particular antenna. Let $\mathcal{V}(\mathbf{Z})$ denote the vector obtained by stacking the columns of the matrix $\mathbf{Z}$. We can express the relation between the compressed measurement vector $\mathbf{y} \in \mathbb{C}^{K}$ and the full matrix $\mathbf{Z} \in \mathbb{C}^{N \times M}$ as

$$
\mathbf{y}=\boldsymbol{\Phi} \mathcal{V}(\mathbf{Z})
$$

It follows from (7) that

$$
\mathbf{y}=\boldsymbol{\Phi} \mathcal{V}(\mathbf{Z})=\boldsymbol{\Phi} \mathcal{V}\left(\mathbf{Z}^{w}\right)+\boldsymbol{\Phi} \mathcal{V}\left(\mathbf{Z}^{t}\right)+\boldsymbol{\Phi} \mathcal{V}(\mathbf{\Upsilon})
$$

Based on the relation between the collected measurements and the formed image given in (6), the formed image can be obtained by solving the following optimization problem:

$$
\begin{array}{cl}
\underset{\mathbf{Z}^{w}, \mathbf{s}}{\operatorname{minimize}} & \left\|\mathbf{Z}^{w}\right\|_{*}+\lambda\|\mathbf{s}\|_{1} \\
\text { subject to } & \left\|\mathbf{y}-\mathbf{\Phi}\left(\mathcal{V}\left(\mathbf{Z}^{w}\right)+\boldsymbol{\Psi} \mathbf{s}\right)\right\|_{2}^{2} \leq \epsilon .
\end{array}
$$

In Equation (11), the sparsity constraint is now imposed on the scene image $\mathbf{s}$, instead of on the target signal matrix $\mathbf{Z}^{t}$. In implementation, the constrained optimization problem can be written into a Lagrangian regularization form:

$$
\min _{\mathbf{Z}^{w}, \mathbf{s}}\left\|\mathbf{y}-\mathbf{\Phi}\left(\mathcal{V}\left(\mathbf{Z}^{w}\right)+\mathbf{\Psi} \mathbf{s}\right)\right\|_{2}^{2}+\lambda_{w}\left\|\mathbf{Z}^{w}\right\|_{*}+\lambda_{s}\|\mathbf{s}\|_{1},
$$

where $\lambda_{w}$ and $\lambda_{s}$ are the regularization parameters for the low-rank and sparse components, respectively.

Next, we introduce an iterative algorithm for solving Problem (12) that comprises a singular value soft-thresholding (SVT) operator used to estimate the low-rank component and an $\ell_{1}$-norm minimization step employed to estimate the sparse vector. Defining a shrinkage operator as $\mathcal{T}_{\tau}(x)=\frac{x}{|x|} \max (|x|-\tau, 0)$, in which $\tau$ is a real value, we express the singular value soft-thresholding operator as

$$
\mathcal{S}_{\tau}\left(\mathbf{Z}^{w}\right)=\mathbf{U} \mathcal{T}_{\tau}(\boldsymbol{\Lambda}) \mathbf{V}^{H},
$$

where $\mathbf{Z}^{w}=\mathbf{U} \boldsymbol{\Lambda} \mathbf{V}^{H}$ is the singular value decomposition of $\mathbf{Z}^{w}$. In the $\ell_{1}$-norm minimization step, a matching pursuit technique is used to obtain a sparse vector for $\mathbf{s}$. The iterative algorithm for solving (12) can be summarized by the following steps:

1) Initialization: Set $\mathbf{Z}_{0}=\mathcal{V}^{*}\left(\boldsymbol{\Phi}^{\dagger} \mathbf{y}\right)$, where $\mathcal{V}^{*}(\cdot)$ denotes the adjoint operator reshaping an $N M \times 1$ column vector to an $N \times M$ matrix, ${ }^{\dagger}$ denotes the pseudo-inverse operator, $\mathbf{Z}_{0}^{w}=\mathbf{Z}_{0}, \mathbf{s}_{0}=\mathbf{0}$, and $i=1$.

2) Singular-value soft thresholding:

$$
\mathbf{Z}_{i}^{w}=\mathcal{S}_{\tau}\left(\mathbf{Z}_{i-1}-\mathcal{V}^{*}\left(\Psi \mathbf{s}_{i-1}\right)\right)
$$


3) Estimating the sparse vector:

$$
\mathbf{s}_{i}=\min _{\mathbf{s}}\left\|\boldsymbol{\Phi} \mathcal{V}\left(\mathbf{Z}_{i-1}-\mathbf{Z}_{i}^{w}\right)-\boldsymbol{\Phi} \mathbf{\Psi} \mathbf{s}\right\|_{2}^{2}+\lambda_{s}\|\mathbf{s}\|_{1}
$$

4) Data consistency and iteration:

If

$$
\frac{\left\|\mathbf{Z}_{i}^{w}+\mathcal{V}^{*}\left(\mathbf{\Psi}_{\mathbf{s}_{i}}\right)-\left(\mathbf{Z}_{i-1}^{w}+\mathcal{V}^{*}\left(\boldsymbol{\Psi}_{\mathbf{s}_{i-1}}\right)\right)\right\|_{F}}{\left\|\mathbf{Z}_{i-1}^{w}+\mathcal{V}^{*}\left(\mathbf{\Psi}_{\mathbf{s}_{i-1}}\right)\right\|_{F}}<\delta
$$

terminate the algorithm,

Else,

compute residual: $\mathbf{r}=\boldsymbol{\Phi} \mathcal{V}\left(Z_{i}^{w}\right)+\boldsymbol{\Phi} \boldsymbol{\Psi}_{\mathbf{s}_{i}}-\mathbf{y}$,

update data: $\quad \mathbf{Z}_{i}=\mathbf{Z}_{i}^{w}+\mathcal{V}^{*}\left(\boldsymbol{\Psi}_{\mathbf{s}_{i}}-\boldsymbol{\Phi}^{\dagger} \mathbf{r}\right)$,

$$
i \leftarrow i+1 \text {. }
$$

go to Step 2.

After the iterative algorithm converges to the desired solution, we rearrange the sparse vector $\mathbf{s}$ into a two-dimensional map representing the formed image of the behind-the-wall target scene. The next section evaluates the proposed approach using real radar data and compares it with several existing imaging techniques.

\section{EXPERIMENTAL RESULTS AND ANALYSIS}

In this section, we present the experimental results obtained using real radar data collected from a monostatic stepped-frequency TWR imaging system. Subsection IV-A describes the scene layout and the parameters of the radar system employed for data collection. Subsection IV-B provides results and performance analysis by several imaging methods.

\section{A. Experimental setup}

The proposed approach is evaluated on real radar data acquired with a radar system placed in front of a concrete wall of thickness $0.14 \mathrm{~m}$. A transceiver is moved along the wall to synthesize a 57-element linear array. At each antenna location, a set of 801 monochromatic signals are transmitted, covering a frequency range of $[0.7,3.1] \mathrm{GHz}$. A wide dihedral of height $0.39 \mathrm{~m}$ and width $0.28 \mathrm{~m}$ is placed behind the wall at a standoff distance of $2.1 \mathrm{~m}$. The imaged scene extends from $[0,4] \mathrm{m}$ in downrange and $[-2,2] \mathrm{m}$ in crossrange. Using the TWR system, a full set of 45,657 space-frequency measurements, i.e., (801 frequencies $\times 57$ antennas) can be acquired. Note that for compressed sensing TWR, only a reduced data set is collected by randomly selecting subset of frequencies and antennas.

In the proposed method, the regularization parameters $\tau$ and $\lambda_{s}$ are both set to 0.001 , and the iterative algorithm is terminated when the relative change of the solution is smaller than $\delta=10^{-6}$, see Step 4. The quality of the formed image is measured using the target-toclutter ratio (in $\mathrm{dB}$ ):

$$
\mathrm{TCR}=10 \log _{10}\left(\frac{\frac{1}{N_{t}} \sum_{q \in A_{t}}\left|I_{q}\right|^{2}}{\frac{1}{N_{c}} \sum_{q \in A_{c}}\left|I_{q}\right|^{2}}\right),
$$

where $A_{t}$ is the target region, $A_{c}$ is the clutter region defined as the entire image excluding the target region, $N_{t}$ and $N_{c}$ are the number of pixels in the target and clutter regions, respectively.

\section{B. Experimental results}

Two experiments are conducted using $10 \%$ of the full data volume. In the first experiment, the same frequencies are selected at each antenna, and spatial filtering [16] and the subspace projection method [5] are applied directly to the reduced data set for wall clutter mitigation. In the second experiment, different frequencies are chosen along the antenna array. To apply the wall clutter mitigation methods, an $\ell_{1}$-norm minimization technique is used to recover the missing frequency samples. For comparison, the two-stage CS-based method proposed by Lagunas et al. [13] is tested on the same reduced data sets. In the two-stage CS-based method, the image formation is formulated as an $\ell_{1}$-norm minimization problem, and the compressive sampling matching pursuit (CoSaMP) algorithm [22] is used to obtain the solution.

Figure 1 shows the formed images obtained from the first experiment. Figure 1(a) illustrates the image formed using CoSaMP, without wall clutter mitigation. This image consists of strong wall clutter, rendering the target detection very difficult. Figures 1(b) and (c) present images obtained from the two-stage CS-based method using spatial filtering and subspace projection for wall clutter mitigation, respectively. We can observe that the target is detected though clutter is still present. Figure 1(d) shows the image obtained from the proposed method. This image has less wall clutter than those produced by the two-stage CS-based method.

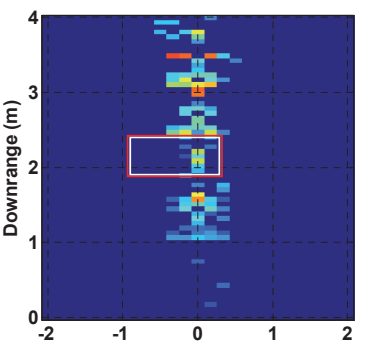

(a)

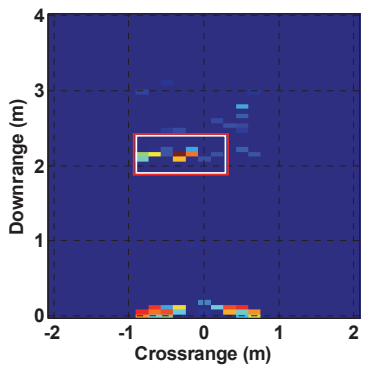

(c)

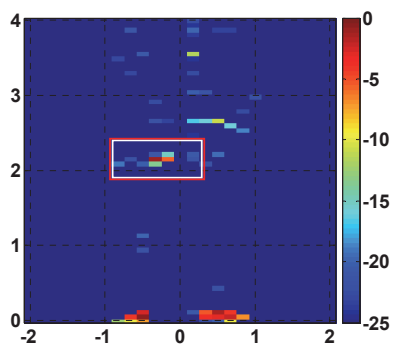

(b)

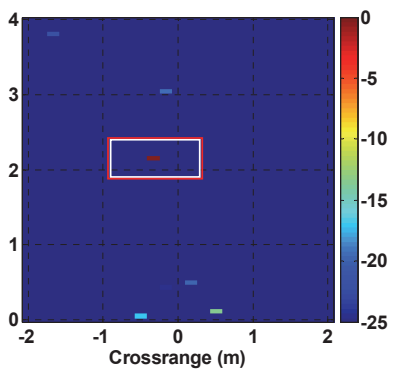

(d)
Fig. 1. Images formed using the same set of frequencies along the antenna array using different image reconstruction and wall clutter mitigation methods: (a) the CoSaMP algorithm without wall clutter mitigation, (b) the two-stage CS-based method in conjunction with spatial filtering, (c) the two-stage CSbased method in conjunction with the subspace projection method, and (d) the proposed joint low-rank and sparsity-based method. Target region is indicated with a solid rectangle.

Figure 2 depicts images obtained from the second experiment. In this experiment, the two-stage CS-based image formation method cannot reveal the target, see Figs. 2(b)-(c). This is due to the low accuracy of the signal recovery technique in estimating the missing frequency measurements. By contrast, the proposed method detects the target well, as illustrated in Fig. 2(d). The reason is that the proposed approach jointly segregates the target signal from the wall reflections and guarantees the sparsity of the scene reconstruction.

In terms of TCR, Table I shows that the proposed method achieves better reconstruction performance than the two-stage CSbased method. The proposed method obtains a TCR of $29.45 \mathrm{~dB}$ for the first experiment and $22.83 \mathrm{~dB}$ for the second experiment. The twostage CS-based method achieves TCRs of $15.51 \mathrm{~dB}$ and $18.50 \mathrm{~dB}$, respectively, when using spatial filtering and subspace projection for removing the wall clutter in the first experiment. It fails to detect the targets in the second experiment. 


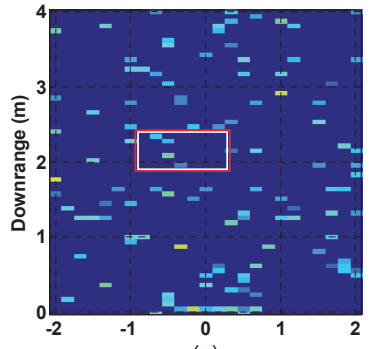

(a)

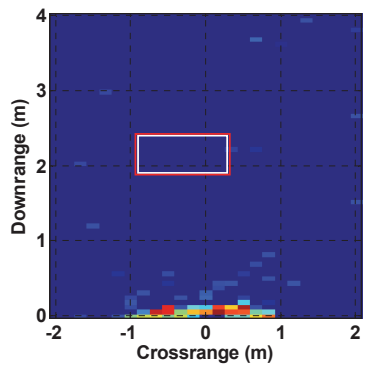

(c)

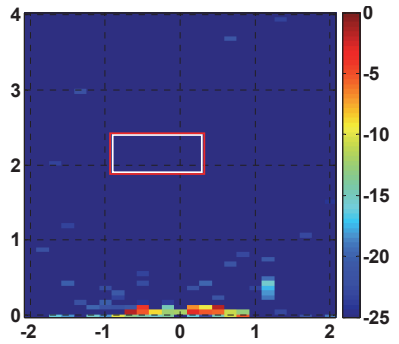

(b)

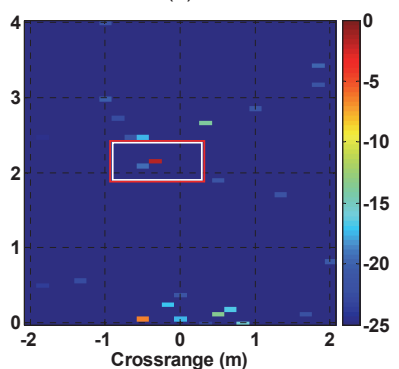

(d)

Fig. 2. Images formed using different sets of frequencies along the antenna array by applying different image reconstruction and wall clutter mitigation methods: (a) the CoSaMP algorithm without wall clutter mitigation, (b) the two-stage CS-based method in conjunction with spatial filtering, (c) the twostage CS-based method in conjunction with the subspace projection method, and (d) the proposed joint low-rank and sparsity-based method.

TABLE I

TARGET-TO-CLUTTER RATIO TCR OF THE SCENE IMAGES FORMED BY DIFFERENT IMAGE FORMATION METHODS.

\begin{tabular}{|l|c|c|}
\hline \hline Scene reconstruction methods & Exp. 1 & Exp. 2 \\
\hline Proposed joint low-rank and sparsity constraints & $\mathbf{2 9 . 4 5}$ & $\mathbf{2 2 . 8 3}$ \\
\hline CS-based method with spatial filtering & 15.51 & 0.0 \\
\hline CS-based method with subspace projection & 18.50 & 0.0 \\
\hline \hline
\end{tabular}

\section{CONCLUSION}

This paper presented a joint low-rank and sparsity-based method for removing wall clutter and forming the image of an indoor scene, using through-the-wall radar imaging. A constrained optimization model is formulated where the nuclear-norm is used to capture the wall returns as a low-rank matrix and the sparsity constraint is imposed to reconstruct the scene as a sparse matrix. An iterative algorithm is developed to jointly estimate the low-rank and sparse matrices. Experimental results show that the proposed approach enhances the target-to-clutter ratio and improves target detection even when the number of measurements is significantly reduced.

\section{ACKNOWLEDGMENTS}

The authors would like to thank Dr. Moeness G. Amin and Dr. Fauzia Ahmad from the Center of Advanced Communications at Villanova University, Villanova, PA, USA, for providing the experimental data. This work is supported by a grant from the Australian Research Council (ARC).

\section{REFERENCES}

[1] M. G. Amin (Ed.), Through-The-Wall Radar Imaging. Boca Raton, FL: CRC Press, 2010.

[2] C. H. Seng, A. Bouzerdoum, M. G. Amin, and S. L. Phung, "Probabilistic fuzzy image fusion approach for radar through wall sensing," IEEE Trans. Image Processing, vol. 22, no. 12, pp. 4938-4951, Dec. 2013.

[3] F. Ahmad and M. G. Amin, "Partially sparse reconstruction of behindthe-wall scenes," Proc. SPIE Compressive Sensing, pp. 83650W.183 650W.9, Maryland, USA, 23-27 Apr. 2012.

[4] M. G. Amin (Ed.), Compressive Sensing for Urban Radar. Boca Raton, FL: CRC Press, 2014.

[5] F. H. C. Tivive, A. Bouzerdoum, and M. G. Amin, "An SVD-based approach for mitigating wall reflections in through-the-wall radar imaging," Proc. IEEE Radar Conf., pp. 519-524, Kansas City, MO, 23-27 May 2011

[6] F. Ahmad, M. G. Amin, and S. A. Kassam, "Synthetic aperture beamformer for imaging through a dielectric wall," IEEE Trans. Aerospace and Electronic Systems, vol. 41, no. 1, pp. 271-283, Jan. 2005.

[7] D. L. Donoho, "Compressed sensing," IEEE Trans. Information Theory, vol. 52, no. 4, pp. 1289-1306, Apr. 2006.

[8] E. J. Candes, J. Romberg, and T. Tao, "Stable signal recovery from incomplete and inaccurate measurements," Communications on Pure and Applied Mathematics, vol. 59, no. 8, pp. 1207-1223, Aug. 2006.

[9] Q. Huang, L. Qu, B. Wu, and G. Fang, "UWB through-wall imaging based on compressive sensing," IEEE Trans. Geoscience and Remote Sensing, vol. 48, no. 3, pp. 1408-1415, Mar. 2010.

[10] Y.-S. Yoon and M. G. Amin, "Through-the-wall radar imaging using compressive sensing along temporal frequency domain," Proc. IEEE Int. Conf. Acoustics, Speech, and Signal Processing, pp. 2806-2809, Dallas, TX, 14-19 Mar. 2010.

[11] M. Leigsnering, C. Debes, and A. M. Zoubir, "Compressive sensing in through-the-wall radar imaging," Proc. IEEE Int. Conf. Acoustics, Speech, and Signal Processing, pp. 4008-4011, Prague, Czech Republic, 22-27 May 2011.

[12] V. H. Tang, A. Bouzerdoum, and S. L. Phung, "Two-stage throughthe-wall radar image formation using compressive sensing," Journal of Electronic Imaging, vol. 22, no. 2, pp. 021 006.1-021 006.10, Apr.-Jun. 2013.

[13] E. Lagunas, M. G. Amin, F. Ahmad, and M. Najar, "Joint wall mitigation and compressive sensing for indoor image reconstruction," IEEE Trans. Geoscience and Remote Sensing, vol. 51, no. 2, pp. 891 - 906, Feb. 2013.

[14] V. H. Tang, A. Bouzerdoum, S. L. Phung, and F. H. C. Tivive, "Enhanced wall clutter mitigation for through-the-wall radar imaging using joint Bayesian sparse signal recovery," Proc. IEEE Int. Conf. Acoustics, Speech and Signal Processing, pp. 7804-7808, Florence, Italy, 4-9 May 2014.

[15] F. Ahmad, J. Qian, and M. G. Amin, "Wall clutter mitigation using discrete prolate spheroidal sequences for sparse reconstruction of indoor stationary scenes," IEEE Trans. Geoscience and Remote Sensing, vol. 53 , no. 3, pp. 1549-1557, March 2015.

[16] Y.-S. Yoon and M. G. Amin, "Spatial filtering for wall-clutter mitigation in through-the-wall radar imaging," IEEE Trans. Geoscience and Remote Sensing, vol. 47, no. 9, pp. 3192-3208, Sept. 2009.

[17] F. H. C. Tivive and A. Bouzerdoum, "An improved SVD-based wall clutter mitigation method for through-the-wall radar imaging," Proc. IEEE Workshop on Signal Processing Advances in Wireless Communications, pp. 430-434, Darmstadt, Germany, 16-19 June 2013.

[18] F. H. C. Tivive, A. Bouzerdoum, and M. G. Amin, "A subspace projection approach for wall clutter mitigation in through-the-wall radar imaging," IEEE Trans. Geoscience and Remote Sensing, vol. 53, no. 4, pp. 2108-2122, Apr. 2015.

[19] A. Y. Aravkin, S. Becker, V. Cevher, and P. A. Olsen, "A variational approach to stable principal component pursuit," in Proc. Conf. Uncertainty in Artificial Intelligence, Quebec, Canada, 23-27 July 2014, pp. $32-41$.

[20] V. Chandrasekaran, S. Sanghavi, P. A. Parrilo, and A. S. Willsky, "Rank-sparsity incoherence for matrix decomposition," SIAM Journal on Optimization, vol. 21, no. 2, pp. 572-596, June 2011.

[21] E. W. Andrew, C. S. Aswin, and R. Baraniuk, "SpaRCS: Recovering low-rank and sparse matrices from compressive measurements," Proc. Advances in Neural Information Processing Systems, pp. 1089-1097, Granada, Spain, 12-17 Dec. 2011.

[22] D. Needell and J. A. Tropp, "CoSaMP: Iterative signal recovery from incomplete and inaccurate samples," Applied and Computational Harmonic Analysis, vol. 26, no. 3, pp. 301-321, May 2009. 